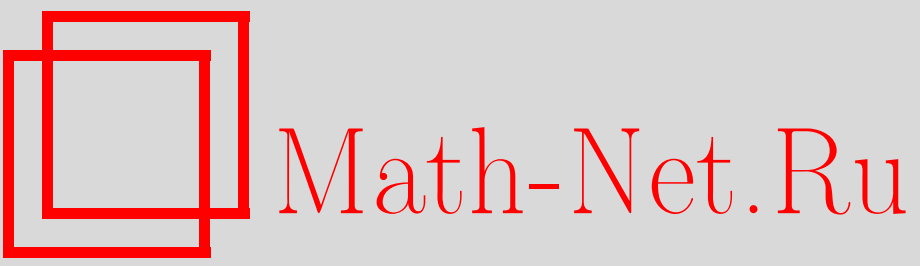

А. М. Олевский, Представление функций экспонентами с положительными частотами, УМН, 2004, том 59, выпуск 1, 169-178

DOI: https://doi.org/10.4213/rm707

Использование Общероссийского математического портала Math-Net.Ru подразумевает, что вы прочитали и согласны с пользовательским соглашением

http://www.mathnet.ru/rus/agreement

Параметры загрузки:

IP: 54.196 .121 .252

26 апреля 2023 г., 04:09:07 


\section{ПРЕДСТАВЛЕНИЕ ФУНКЦИЙ ЭКСПОНЕНТАМИ С ПОЛОЖИТЕЛЬНЫМИ ЧАСТОТАМИ}

А. М. ОлЕВСКИй

Мы обсуждаем некоторые новые аспекты следующей общей проблемы: какие спектры частот позволяют представить произвольную функцию в виде суммы ряда соответствующих гармоник.

Нас в особенности интересует "аналитическая" ситуация, когда задействованы лишь положительные частоты.

Библиография: 30 названий

\section{СОДЕРЖАНИЕ}

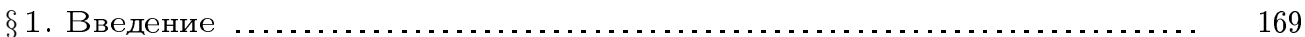

$\S 2$. Редкие спектры Меньшова ........................................ 170

$\S 3$. Положительный спектр: представление по мере . . . . . . . . . . . . . . . 172

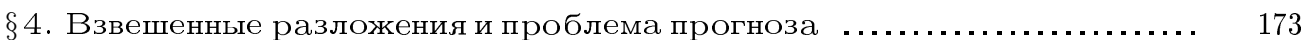

$\S 5$. Поточечные аналитические разложения $\ldots \ldots \ldots \ldots \ldots \ldots \ldots \ldots \ldots \ldots \ldots . . \ldots \ldots$

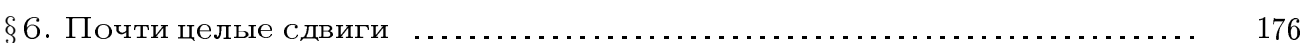

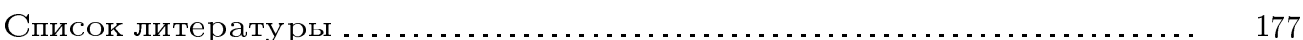

\section{$\S$ 1. Введение}

1.1. Проблемы гармонического анализа на групе окружности $\mathbb{T}$ требуют экспонент с цельми частотами (= двойственная группа). Аналогично, на $\mathbb{R}$ используются все действительные частоты. Однако в некоторых случаях лишш часть "стандартного" спектра частот достаточна для решения проблемы. Мы намерены обсудить некоторые недавние результаты в этом направлении. Нас в особенности интересует "аналитическая" ситуация, когда задействованы лишь положительные частоты.

1.2. Классический пример такой ситуации доставляет теорема Колмогорова-Сегё. Рассмотрим весовую аппроксимацию на $\mathbb{T}$ посредством тригонометрических полиномов. Если вес $w$ отделен от нуля, то, разумеется, удаляя какую-либо из экспонент, $\left\{e^{i n t}, n \in \mathbb{Z}\right\}$, мы теряем полноту. Однако если вес имеет “глубокий” нуль, то экспоненты с положительными частотами полны в пространстве $L^{2}(w, \mathbb{T})$. Точнее, для этого необходима и достаточна расходимость логарифмического интеграла:

$$
\int_{\mathbb{T}} \log w(t) d t=-\infty
$$


На вероятностном языке это условие описьвает ситуацию, когда "будущее" допускает точное предсказание [1], [2].

Мы рассмотрим следуюшую проблему: возможно ли более сильное свойство, когда любая функция $f \in L^{2}(w, \mathbb{T})$ представима рядом

$$
f=\sum_{n>0} c(n) e^{i n t},
$$

сходяшимся по норме? Оказывается, что для весов общего положения ответ положительный. В $\S 4$ мы обсудим этот результат в контексте колмогоровской теории экстраполяции. Близкий вопрос о разложении произвольной функции в ряд (2), сходящийся по мере, рассматривается в $\S 3$.

1.3. Нас интересует также поточечное представление функций тригонометрическими рядами. Фундаментальньй результат в этом направлении принадлежит Д.Е. Меньшову [3], см. также [4]: каждая функция класса $L^{0}(\mathbb{T})$, т.е. измеримая функция $f: \mathbb{T} \rightarrow \mathbb{C}$, допускает разложение в ряд

$$
f=\sum_{n \in \mathbb{Z}} c(n) e^{i n t},
$$

сходяшийся почти всюду (п.в.).

Это, вообще говоря, не ряд Фурье, поскольку не предполагается интегрируемость функции ни в каком смысле. Но даже если $f \in L^{1}$ и ряд Фурье-Лебега определен, он не обязан сходиться поточечно, как показывает знаменитый пример Колмогорова [5].

Следует отметить, что разложение (3) существенно неединственно. Коэффициенты $\{c(n)\}$ определяются посредством деликатной, но достаточно свободной процедуры, позволяюшей исключить любое конечное и даже некоторые бесконечные множества гармоник.

Какой спектр частот на самом деле необходим для представления произвольной функции? Мы обсудим этот вопрос в $§ 2$. Там же рассматривается непериодическая версия.

Как и выше, мы специально интересуемся "аналитическим" представлением (2). Основной результат $\S 5$ описьвает новый, и, быть может, неожиданный, эффект, проявляюшийся в этой ситуации.

1.4. Несколько иная, но на самом деле тесно связанная с предыдущим проблема рассматривается в $\S 6$ : каковы “спектры сдвигов", порождающие все пространство $L^{2}(\mathbb{R})$, исходя из единой функции (генератора)? И может ли этот генератор обладать хорошей "пространственно-частотной" локализацией?

1.5. Содержание $\S \S 2-5$ основано на работах $[6],[7]$ и результатах, полученных совместно с Г. Козма [8]-[11]. В $\S 6$ формулируются результаты из [6] и совместной с А. Улановским работы [12].

\section{§ 2. Редкие спектры Меньшова}

2.1. Представление на окружности. Введем следуюшее

ОПредЕЛЕниЕ. Множество $\Lambda \subset \mathbb{Z}$ назьвается спектром Меньшова, если каждая функция $f \in L^{0}(\mathbb{T})$ допускает представление

$$
f=\sum_{n \in \Lambda} c(n) e^{i n t},
$$

где ряд сходится п.в. 
Нас интересует, насколько редким может быть спектр Меньшова. Ф.Г. Арутюнян доказал [13], что множество $\Lambda$, симметричное относительно нуля и содержашее сколь угодно длинные отрезки целых чисел, является спектром Меньшова. Наш результат показывает, что спектр Меньшова может быть лакунарным, т.е. удовлетворять условию

$$
\lambda(n+1)-\lambda(n) \rightarrow \infty \quad(n \rightarrow \infty) .
$$

Более того, лакуны могут расти “почти” в геометрической прогрессии:

Теорема 2.1. Для заданной последовательности $0<\varepsilon(n)=o(1)$ можноо построить симметричный спектр Меньшова такой, что

$$
\lambda(n+1) / \lambda(n)>1+\varepsilon(n) \quad(n=1,2, \ldots) .
$$

Точность этого условия вытекает из классических результатов об адамаровской лакунарности.

Однако не только метрические свойства спектра важны в задаче представления функций. Локализация лакун тоже играет важную роль. Например, $\Lambda=\left\{ \pm k^{2}\right\}$ не является спектром Меньшова. Это несложно вьвести из того факта, что $k^{2}+1$ не делится на 3 . Несколько более деликатные аргументы такого сорта показывают, что утверждение сохраняется при ограниченных возмушениях квадратов. С другой стороны, справедливо следуюшее предложение.

ТЕОРема 2.2. Для любой последовательности $w(k) \nearrow \infty$ существует симметричный спектр Меньшова

$$
\Lambda=\left\{ \pm k^{2}+o(w(k))\right\}
$$

Сформулированные теоремы были установлены в [9]. Доказательства используют альтернативную версию метода Меньшова, указанную нами в [14]. Позднее аналогичньй подход был независимо развит Т. В. Кёрнером [15].

Наша конструкция разреженных спектров Меньшова использует "блоки", насыщенные конечными арифметическими прогрессиями. Доказательство теоремы 2.2 труднее, поскольку возможности конструкции таких блоков вблизи квадратов ограничены. Нам неизвестно, справедлив ли аналог последней теоремы для кубов.

2.2. Непериодический случай. По аналогии с интегралом Фурье можно ожидать, что для функщий на оси представление (3) преврашается в “тригонометрический интеграл", используюший все действительные частоты. Такая версия теоремы Меньшова была доказана Р. С. Давтяном [16]. С другой стороны, предыдущие результаты дают основание предположить, что можно обойтись значительно меньшим спектром частот. Это в самом деле так: сушествуют дискретные спектры, доставляющие поточечные разложения произвольных измеримых функций в ряды экспонент.

Вначале сформулируем теорему об аппроксимации.

ТеОРема 2.3 [6]. Пусть $\Lambda$ - “почти цельй спектр, т.е. последовательность

$$
\lambda(n)=n+\alpha(n), \quad n \in \mathbb{Z}, \quad 0<|\alpha(n)|=o(1) \quad(n \rightarrow \pm \infty) .
$$

Тогда система

$$
\left\{e^{i \lambda t}\right\}_{\lambda \in \Lambda}
$$

полна в $L^{0}(\mathbb{R})$ по отношению $к$ сходимости п.в. 
Нет необходимости возмущать все целые числа. На самом деле частоты, обеспечиваюшие данное свойство полноты, могут расти "почти" в геометрической прогрессии, как в [3] (см. [17]).

Доказательство теоремы 2.3 опирается на конструкцию, используюшую "малые знаменатели". Комбинируя эту теорему с техникой меньшовского типа, можно доказать следующее предложение.

ТеОРема 2.4 [8]. Существует спектр (5), позволяющий представить любую функцию $f \in L^{0}(\mathbb{R})$ в виде ряда

$$
f=\sum_{\lambda \in \Lambda} c_{\lambda} e^{i \lambda t} \quad\left(=\lim _{N \rightarrow \infty} \sum_{|\lambda|<N}\right),
$$

сходящегося п.в.

Однако, в противоположность теореме 2.3 , не каждьй “почти целый” спектр (5) доставляет указанное представление. В частности, возмушения $\alpha(n)$ не могут убьвать слишком быстро. Тем не менее, свойство, сформулированное в последней теореме, не является исключительным. Оно появляется при случайны $x$ возмушениях целых частот.

ТЕОРема $2.5[10]$. Пусть $\{\alpha(n)\}$ последовательность независимых случайных величин, равномерно распределенных на отрезке $[-d, d]$ (или на отрезках $[-d(n), d(n)]$, где $d(n)$ стремится $\kappa$ нулю достаточно медленно). Тогда $c$ вероятностью 1 спектр $\Lambda=\{n+\alpha(n)\}$ удовлетворяет заключению предьцдущей теоремы.

\section{§ 3. Положительный спектр: представление по мере}

Каковы возможности представления экспонентами с положительньми частотами? $\mathrm{C}$ точки зрения размера и арифметики множество $\mathbb{Z}^{+}$вьглядит вполне удовлетворительно. С другой стороны, ряд (2) - это степенной ряд

$$
\sum c(n) z^{n}
$$

на окружности $|z|=1$. Поэтому можно ожидать, что функция, представимая этим рядом, несет в себе следы аналитичности. И в самом деле, пусть ряд сходится в точке $z_{0}$ единичной окружности к значению $A$. Тогда согласно классической теореме Абеля аналитическая функция $F(z)$, определенная в круге посредством ряда (8), имеет угловое граничное значение в точке $z_{0}$, равное $A$. Теперь используем замечательную теорему единственности Привалова (1919, см. [18]): если аналитическая в круге функция $F$ имеет некасательньй предел 0 на множестве положительной меры на границе, то $F \equiv 0$. Отсюда, в частности, следует, что $\mathbb{Z}^{+}$не является спектром Меньшова. Более того, функция $f \not \equiv 0$, поточечно представимая рядом (2), должна быть квазианалитичной в том смысле, что она не может обрашаться в нуль на множестве положительной меры.

Иногда сходимость по мере более эффективна в проблеме представления, чем поточечная сходимость. Впервые это было доказано Д.Е. Меньшовым [19] при разложении функций, принимаюших бесконечные значения. Затем А. А. Талалян [20] использовал сходимость по мере для представления функций рядами по полным ортогональным системам. В обоих случаях поточечная сходимость не работает. Это было доказано соответственно С. В. Конягиным [21] и Б. С. Кашиным [22]. 
Оказьвается, что и в "аналитической" ситуации сходимость по мере (т.е. в метрике пространства $L^{0}$ ) позволяет получить теорему представления без каких-либо дополнительных ограничений.

Теорема 3.1 [9]. Каждая измеримая функиия $f$ на окружности представима рядом (2), сходящимся по мере.

При этом нет необходимости использовать все положительные частоты; как и ранее, можно обойтись достаточно редким спектром. Верны соответствующие аналоги сформулированных выше теорем о "почти адамаровском" спектре и о "возмущенных квадратах". Первый из них получается комбинацией методов, использованных при доказательстве теорем 2.1 и 3.1. Второй результат требует новых соображений, см. [9].

ЗАмечАниЕ. Назовем $\Lambda \subset \mathbb{Z}$ спектром аппроксимации, если любая измеримая функция на $\mathbb{T}$ представима как предел сходящейся п.в. последовательности тригонометрических полиномов с частотами, принадлежашими $\Lambda$. Это - более слабое понятие, чем спектр Меньшова. Пример: $\Lambda=\mathbb{Z}$.

Вероятно, спектр аппроксимации не всегда позволяет даже получить разложение по мере; однако мы не располагаем соответствуюшим примером.

\section{§4. Взвешенные разложения и проблема прогноза}

Имея разложение по мере, можно пытаться получить более сильное разложение в весовом пространстве.

Напомним, что условие Колмогорова-Сегё (1) ответственно за полноту системы $\left\{e^{i n t}\right\}_{n>0}$ в $L^{2}(w, \mathbb{T})$. В рамках колмогоровской теории экстраполяции [1], [2] это означает следующее: если спектральная плотность $w$ стационарного случайного процесса $\{X(n)\}, n \in \mathbb{Z}$, удовлетворяет условию (1), и только в этом случае, можно в точности предсказать будушее по прошлому. Таким образом, $X(0)$ может быть записано с произвольно малой ошибкой $\varepsilon$ в виде конечной линейной комбинации

$$
X(0) \approx \sum_{n>0} c(n) X(-n) .
$$

Коэффициенты зависят от $\varepsilon$ и берутся из условия весовой аппроксимации функции $f=1$ :

$$
\left\|1-\sum_{n>0} c(n) e^{i n t}\right\|<\varepsilon .
$$

Предположим теперь, что "прошлое" $X(-n)$ известно с некоторой погрешностью. Тогда, чтобы избежать влияния этого "шума" на прогноз, естественно потребовать, чтобы коэффициенты $c(n)$ были малы или, по крайней мере, ограничены константой, не зависяшей от $\varepsilon$ :

$$
|c(n ; \varepsilon)|<A .
$$

Однако условие (1) не обеспечивает подобной аппроксимации. Более того, несложная оценка гармонической меры показьвает, что такая аппроксимация невозможна, если вес $w$ отделен от нуля на какой-нибудь дуге, см. [7]. 
С другой стороны, требуемая аппроксимация следовала бы немедленно из разложения

$$
1=\sum_{n>0} c(n) e^{i n t}
$$

сходящегося в $L^{2}(w, \mathbb{T})$.

Возможно ли такое разложение для нетривиального веса? Препятствие обозначено неравенством Иенсена, из которого вытекает, что частичные суммы ряда (9) должны быть экспоненциально велики на некоторых множествах малой меры. К счастью, техника, использованная при доказательстве теоремы 3.1 , позволяет контролировать локализацию этих "сверхбольших" значений одновременно для всех частичных сумм и подавить их соответствующим выбором весовой функции $w$.

Теорема 4.1 [7]. Существует вес $w>0$ п.в. такой, что любая функиия $f \in$ $L^{2}(w, \mathbb{T})$ допускает разложсение в ряд $(2)$, сходящийся по норме.

Как отмечено выше, функция $w$ не может вести себя регулярно. В частности, она с необходимостью разрьвна почти всюду. Однако иррегулярность нередко является “типичньм" явлением, так что a priori не исключено, что для большинства весов разложение (9) возможно. Оказьвается, что это действительно так.

Чтобы сформулировать результат, рассмотрим метрическое пространство весов

$$
W=\{w: 0 \leqslant w(t) \leqslant 1\}, \quad \rho\left(w, w^{\prime}\right):=\int_{\mathbb{T}}\left|w-w^{\prime}\right| d t
$$

Это - полное пространство, и мы можем использовать бэровские категории для определения общего положения.

Теорема 4.2 [7]. Свойство веса, сформулированное в теореме 4.1, - типично, т.е. справедливо для всех $w$, за исключением множества первой категории.

\section{$\S 5$. Поточечные аналитические разложения}

5.1. Вернемся к представлениям (2), сходяшимся почти всюду. Кардинальное отличие от ситуации, рассмотренной в $\S \S 2-4$, состоит в том, что каждая функция допускает не более одного такого разложения. Это следует из теорем Абеля и Привалова.

Обычно, если представление посредством гармоник единственно, то коэффициенты восстанавливаются по формулам Фурье. Сравним, например, классические результаты о сходимости всюду: если ряд (3) представляет нуль, то $c(n)=0$ для всех $n$ (Кантор, 1872); и сильнее: если этот ряд представляет $L^{1}$-функцию $f$, то он является рядом Фурье (Дюбуа-Реймон, 1876, и Валле-Пуссен, 1911). В более общей форме этот принцип установлен И.И. Приваловым [23]: если ряд (3) сходится к $f \in L^{1}(\mathbb{T})$ в каждой точке вне некоторого замкнутого множества единственности $K$, то он является рядом Фурье-Лебега функции $f$.

Учитывая свойство единственности аналитических разложений, естественно ожидать, что если ряд (2) сходится почти всюду к интегрируемой функции $f$, то он является рядом Фурье.

Оказьвается, это не так. 
Tеорема 5.1 [11]. Существует степенной ряд (8), сходящийся почти всюду на окружности $|z|=1 \kappa$ функиии $f \in L^{2}(\mathbb{T})$, но не принадлежсащий пространстве Харди $H^{2}(D)$.

Это означает, что функция $f$, помимо классического разложения Фурье, сходящегося п.в. в силу теоремы Карлесона и имеющего непустую отрицательную часть спектра, допускает еще одно - также единственное - разложение по гармоникам с положительными частотами.

Доказательство теоремы основано на конструкции вероятностной меры $\mu$ с компактньм носителем $K \subset \mathbb{T}$ лебеговой меры нуль такой, что функция

$$
F(z)=\exp \left(\int_{\mathbb{T}} \frac{e^{i t}+z}{e^{i t}-z} d \mu(t)\right)
$$

имеет коэффищиенты Тейлора, стремяшиеся к нулю. Ряд Тейлора такой меры представляет "аналитическую псевдофункцию” на окружности, и классическая риманова теория влечет сходимость вне $K$ к функции $F\left(e^{i t}\right) \in L^{\infty}$. Однако $F$, будучи неограничена внутри круга, не является интегралом Пуассона от своих граничных значений.

Отметим, что компакт $K$ обязан быть “толстым": иметь хаусдорфову размерность 1. В самом деле, из теоремы типа Фрагмена-Линделёфа для функций медленного роста в круге (см. [24], [25]) легко следует, что если ряд (2) сходится к функиии $f \in L^{1}(\mathbb{T})$ вне множества $E, \operatorname{dim}_{H} E<1$, то он является рядом Фурье.

5.2. Теорема 5.1 позволяет по-новому взглянуть на свойства так называемых нульрядов, т.е. (нетривиальных) рядов (3), сходящихся к нулю п.в. Впервые пример такого рода был построен Д.Е. Меньшовым (1916) в качестве решения проблемы, поставленной Н. Н. Лузиным в его фундаментальной диссертации (1915) (см. [26]). Этот результат положил начало современной теории единственности в анализе Фурье. В дальнейшем он развивался в различных направлениях, см. [4], [27]. В частности, было показано, что коэффициенты нуль-ряда могут стремиться к нулю настолько быстро, насколько это возможно в рамках необходимого условия: $\{c(n)\} \notin l_{2}$. Наиболее сильные результаты здесь принадлежат О.С. Ивашеву-Мусатову, Н.Б. Погосяну, Ф. Г. Арутюняну и Т.В. Кёрнеру.

С другой стороны, по-видимому, во всех известных конструкциях амплитуды $\{|c(n)|\}$ в положительной и отрищательной частях спектра ведут себя одинаково. Наш результат показьвает, что возможна существенная асимметрия: одна из частей ряда, аналитическая или антианалитическая, может принадлежать $L^{2}(\mathbb{T})$.

Теорема 5.2 [11]. Существует нуль-ряд (3) с условием

$$
\sum_{n<0}|c(n)|^{2}<\infty
$$

В самом деле, возьмем $f$ из теоремы 5.1 и рассмотрим разность между ее рядом Фурье и "неклассическим" разложением (2).

5.3. В еще не опубликованной работе, совместной с Г. Козма, нами доказано, что эффект, установленный в теореме 5.1, возможен даже для бесконечно гладких функций $f$. Таким образом, амплитуды антианалитической части нуль-ряда могут убывать быстрее любой степени. Нам удалось точно описать возможную скорость убывания. Она характеризует новый тип квазианалитичности, сушественно отличньй от классического. 


\section{$\S$ 6. Почти целые сдвиги}

6.1. Винер доказал, что если преобразование Фурье функции $\varphi$ не обращается в нуль, то множество всех ее сдвигов полно. Иногда даже дискретное семейство сдвигов обладает этим свойством.

ОПРЕДЕЛЕНИЕ. Скажем, что спектр $\Lambda \subset \mathbb{R}$ допускает генератор $\varphi \in L^{2}(\mathbb{R})$, если семейство сдвигов

$$
\{\varphi(t-\lambda)\}_{\lambda \in \Lambda}
$$

порождает пространство $L^{2}(\mathbb{R})$.

Как много сдвигов необходимо для этого?

Из теории целых функций следует, что если $\Lambda$ имеет бесконечную верхнюю плотность, т.е.

$$
\lim \sup \frac{1}{r} \operatorname{Card}(\Lambda \cap[-r, r])=\infty,
$$

то генератор существует [28]. С другой стороны, из соображений периодичности легко видеть, что $\Lambda=\mathbb{Z}$ не допускает генератора. Возникает вопрос: размер спектра или его арифметика ответственны за рассматриваемое свойство? В частности, что происходит при малых возмущениях целых чисел?

Ответ на этот вопрос был получен нами в [6]:

ТЕОРЕМа 6.1. Каждый "почти иельй” спектр (5) (даже положительная его часть) допускает генератор.

Следующее замечание показьвает тесную связь задачи с основным содержанием данной работы: $\Lambda$ допускает генератор если и только если семейство әкспонент $(6)$ полно в $L^{0}(\mathbb{R})$ относительно сходимости почти всюду.

Если последнее свойство вьполнено, то несложно определить суммируемый положительньй вес $w$ на $\mathbb{R}$ такой, что семейство (6) полно в $L^{2}(w, \mathbb{R}) ;$ далее - преобразование Фурье задает генератор:

$$
\varphi=\widehat{\sqrt{w}} .
$$

Таким образом, теорема 6.1 является следствием теоремы 2.3 .

6.2. Следует заметить, что конструкция [6], используюшая “малые знаменатели", позволяет построить генератор гладкий (даже целую функцию), но медленно убывающий на бесконечности.

Можно ли построить генератор $\varphi$, принадлежаший пространству Шварца $S$ ? Оказывается, это зависит от индивидуальных свойств спектра.

Напомним, что Г. Ландау [29] указал специальную последовательность малых возмущений $\{\alpha(n)\}$ такую, что система (6) полна в $L^{2}$ на любом множестве вида:

$$
[-A, A] \backslash \bigcup_{k \in \mathbb{Z}}(2 \pi k-\varepsilon, 2 \pi k+\varepsilon)
$$

А. Улановский [30] распространил этот результат на случай экспоненциально убываюших возмушений. Заметим, что, имея полноту на "множествах Ландау", несложно построить генератор $\varphi \in S$.

Удивительно, что если возмущения убывают не так быстро (т.е. мы находимся дальше от критического случая $\Lambda=\mathbb{Z}$ ), то “хороший" генератор может не сушествовать. Точная количественная оценка содержится в следующей теореме. 
Теорема 6.2 [12]. Рассмотрим возмущения

$$
0<|\alpha(n)|=O\left(e^{-r(n)}\right),
$$

где $\{r(n)\}$ - заданная последовательность (удовлетворяющая некоторым условиям регулярности). Тогда

(i) если $r$ - квазианалитична, т.е.

$$
\sum r(n) / n^{2}=\infty
$$

то спектр $\Lambda=\{n+\alpha(n)\}$ допускает генератор $\varphi \in S$;

(ii) в противном случае существуют возмущения (10) такие, что $\Lambda$ не допускает даже генератора $\varphi \in L^{1}(\mathbb{R})$.

6.3. Возврашаясь к вопросу, насколько редким может быть спектр, допускающий генератор, отметим, что $\lambda(n)$ могут расти “почти в геометрической прогрессии" в смысле (4), см. [17]. Однако адамаровская лакунарность невозможна.

Таким образом, если спектр слишком плотный или слишком редкий, то арифметика его не имеет значения. Однако она становится определяющей в случае промежуточного роста между арифметической и геометрической прогрессиями.

\section{СПИСОК ЛИТЕРАТУРЫ}

[1] А. Н. Колмогоров. Стационарные последовательности в гильбертовском пространстве // Бюл. МГУ. Матем. 1941. Т. 2. № 6. С. 1-40; А. Н. Колмогоров. Теория вероятностей и математическая статистика. М.: Наука, 1986. С. 215-255.

[2] А.Н. Колмогоров. Интерполирование и экстраполирование стационарных случайных последовательностей // Изв. АН СССР. Сер. матем. 1941. Т. 5. № 1. С. 1-40; А. Н. Колмогоров. Теория вероятностей и математическая статистика. М.: Наука, 1986.

[3] D. E. Menchoff. Sur la représentation des fonctions mesurables par des séries trigonometriques // Матем. сб. 1941. Т. 9. № 3. С. 667-692.

[4] Н. К. Бари. Тригонометрические ряды. М.: Физматгиз, 1961.

[5] A. Kolmogoroff. Une série de Fourier-Lebesgue divergente presque partout // Fund. Math. 1923. V. 4. Р. 324-328; Рус. пер.: Ряд Фурье-Лебега, расходящийся почти всюду // А.Н. Колмогоров. Избранные труды. Математика и механика. М.: Наука, 1985. C. 8-11.

[6] A. Olevskii. Completeness in $L^{2}(\mathbb{R})$ of almost integer translates // C. R. Acad. Sci. Paris. Sér. I Math. 1997. V. 324. № 9. P. 987-991.

[7] A. Olevskii. On the "prediction" problem // C. R. Math. Acad. Sci. Paris. 2002. V. 334. № 4. P. 279-282.

[8] G. Kozma, A. Olevskii. Representation of non-periodic functions by trigonometric series with almost integer frequencies // C. R. Acad. Sci. Paris. Sér. I Math. 1999. V. 329. № 4. P. 275-280.

[9] G. Kozma, A. Olevskii. Menshov representation spectra // J. Anal. Math. 2001. V. 84. P. 361-393.

[10] G. Kozma, A. Olevskii. Random Menshov spectra // Proc. Amer. Math. Soc. 2003. V. 131. №6. P. 1901-1906.

[11] G. Kozma, A. Olevskii. A null series with small anti-analytic part // C. R. Math. Acad. Sci. Paris. 2003. V. 336. №6. P. 475-478.

[12] A. Olevskii, A. Ulanovskii. Almost integer translates. Do nice generators exist? // J. Fourier Anal. Appl. (to appear).

[13] Ф. Г. Арутюнян. Представление измеримых функций нескольких переменных тригонометрическими рядами // Матем. сб. 1985. Т. 126. № 2. С. 267-285.

[14] А. М. Олевский. Модификация функций и ряды Фурье // УМН. 1985. Т. 40. № 3. C. $157-193$. 
[15] T. W. Körner. On the representation of functions by trigonometric series // Ann. Fac. Sci. Toulouse Math. (6). 1996. Special issue. P. 77-119.

[16] Р. С. Давтян. Представление измеримых функций тригонометрическими интегралами // Докл. АН АрмССР. 1971. Т. 53. С. 203-207.

[17] A. Olevskii. Approximation by translates on $\mathbb{R} / /$ Real Anal. Exchange. 1998/99. V. 24. № 1. P. 43-44.

[18] И.И. Привалов. Граничные свойства аналитических функций. М.-Л.: ГТТИ, 1950.

[19] Д. Е. Меньшов. О сходимости по мере тригонометрических рядов // Труды МИАН. 1950. Т. 32.

[20] А. А. Талалян. Представление измеримых функций рядами // УМН. 1960. Т. 15. № 5. C. $77-141$.

[21] С. В. Конягин. О пределах неопределенности тригонометрических рядов // Матем. заметки. 1988. Т. 44. №6. С. $770-783$.

[22] Б. С. Кашин. Об одной полной ортонормированной системе // Матем. сб. 1976. Т. 99. C. $356-365$.

[23] И.И. Привалов. Обобщение теоремы Paul du Bois-Reymond'a // Матем. сб. 1923. Т. 31. № 2. C. 229-231.

[24] B. Dalberg. On the radial boundary values of subharmonic functions // Math. Scand. 1977. V. 40. P. 301-307.

[25] R. D. Berman. Boundary limits and an asymptotic Phragmén-Lindelöf theorem for analytic functions of slow growth // Indiana Univ. Math. J. 1992. V. 41. № 2. P. 465-481.

[26] Н. Н. Лузин. Интеграл и тригонометрический ряд. М.-Л.: Гостехиздат, 1951.

[27] J.-P. Kahane, R. Salem. Ensembles parfaits et séries trigonométriques. Paris: Hermann, 1994.

[28] А. М. Седлецкий. Приближение сдвигами и полнота весовой системы экспонент в $L^{2}(\mathbb{R})$ // Матем. сб. 1984. Т. 123. №1. С. 92-107.

[29] H. Landau. A sparse regular sequence of exponentials closed on large sets // Bull. Amer. Math. Soc. 1964. V. 70. P. 566-569.

[30] A. Ulanovskii. Sparse systems of functions closed on large sets in $\mathbb{R}^{N} / / \mathrm{J}$. London Math. Soc. (2). 2001. V. 63. № 2. P. 428-440.

School of Mathematical Sciences,

Поступила в редакцию

Tel Aviv University, Israel

27.11 .2003

E-mail: olevskii@post.tau.ac.il 